\title{
Limited macular translocation
}

\section{Magnitude of the public health problem of age-related macular degeneration}

Subfoveal choroidal neovascularisation (CNV) is a major cause of central vision loss in adults. ${ }^{1,2}$ Estimates from community- and clinic-based studies showed that of the 30 million people in the United States who were 65 years of age or older in 1990, between 150000 and 200000 developed CNV in one or both eyes during the next year. ${ }^{3-5}$ In a review of multiple population-based studies a consistent increase with age was identified in the prevalence of late lesions of age-related macular degeneration (AMD), from near absence at age 50 years to about $2 \%$ prevalence at age 70 years and about $6 \%$ at age 80 years. $^{6}$

Although research has identified some factors associated with or predictive of increased risk of $\mathrm{AMD}^{7-9}$ this condition remains one of the most difficult challenges to vision, and several treatment modalities are under investigation. ${ }^{10-14}$

\section{Treatments for age-related macular degeneration}

The Macular Photocoagulation Study (MPS) showed that, in selected eyes, laser photocoagulation is successful in limiting severe vision loss, but this benefit occurred at the expense of immediately decreasing central vision. ${ }^{15,16}$ Eyes with new subfoveal CNV treated with laser photocoagulation achieved an average visual acuity of 20/320 compared with $20 / 400$ in non-treated eyes after 24 months of follow-up. In addition, the benefits of treatment over the natural history of the condition only became apparent 6 months later and retention or recovery of good vision rarely occurred in treated patients. None of the 77 eyes in the new subfoveal CNV study had a visual acuity of $20 / 80$ or better, while $88 \%$ had a visual acuity of $20 / 200$ or worse at the 48 month examination following laser ablation of the subfoveal lesion. Recurrence or persistence of the CNV was also common following treatment, with close to half of the treated eyes having persistent or recurrent $\mathrm{CNV}$ within 3 years. ${ }^{17}$

The surgical approach to subfoveal lesions was initiated by de Juan and Machemer ${ }^{18}$ and subsequently described by Blinder and colleagues. ${ }^{19}$ The technique initially described relied on the creation of a large circumferential retinotomy around the macula to create a flap that is retracted to expose the subretinal space. Later, Thomas ${ }^{20}$ emphasised the creation of a small retinotomy eccentric to the fovea and the manipulation of the neovascular complex using instruments introduced through this entry site and visualised through neurosensory retina. Using this technique, Thomas and co-workers ${ }^{21}$ reported that of 22 eyes with subfoveal $\mathrm{CNV}$ secondary to AMD that underwent surgical membrane removal, 19 (86\%) achieved a postoperative visual acuity of $20 / 200$ or worse after an average post-operative follow-up of 7.8 months. Clinicopathological features of surgically excised choroidal neovascular membranes suggest that most are sub-retinal pigment epithelial. ${ }^{22}$ Because of this location, by removing the $\mathrm{CNV}$ from eyes with $\mathrm{AMD}$, the retinal pigment epithelium/choriocapillaris complex is likely to be inadvertently and concomitantly removed, limiting the visual improvement. The Submacular Surgery Trials are currently under way to evaluate the exact role of surgical removal of $\mathrm{CNV}$.

More recently, photodynamic therapy with verteporfin has been shown to be beneficial in reducing the risk of vision loss in eyes with predominantly classic CNV secondary to AMD. ${ }^{10}$ The primary outcome at the 12 month examination was that $6 \%$ of the eyes treated with verteporfin compared with $2.4 \%$ of the eyes given placebo had an increase of 3 or more Snellen lines in visual acuity. ${ }^{10}$ While the two groups had a similar mean visual acuity at baseline (approximate Snellen equivalent of $20 / 80^{-2}$ ), by 12 months the mean visual acuity in eyes treated with verteporfin compared with eyes given placebo was $20 / 160^{+2}$ versus $20 / 200$. $^{10}$

The idea of retinal translocation was first presented in $1983 .{ }^{23}$ Initially, the feasibility of retinal translocation was tested in rabbit models. Later, Machemer and Steinhorst ${ }^{24,25}$ reported a technique involving lensectomy and vitrectomy, followed by the infusion of fluid into the subretinal space to achieve retinal separation. A $360^{\circ}$ peripheral retinectomy was then created and the retina rotated by as much as $60^{\circ}$ around the optic nerve and reattached. This procedure was performed on 3 patients with limited but encouraging results. ${ }^{25}$ Of the 3 patients, 1 had significantly improved vision and the others experienced complications. The
E. de Juan

G.Y. Fujii

The Wilmer

Ophthalmological Institute The Johns Hopkins Hospital Baltimore

Maryland, USA

Eugene de Juan Jr, MD The Wilmer

Ophthalmological Institute The Johns Hopkins University

Maumenee Building (Room 721)

600 North Wolfe Street Baltimore MD 21287-9277, USA

Tel: +1 (410) 5025383

Fax: +1 (410) 6148577

e-mail: edejuan@jhmi.edu 
procedure was difficult and lengthy but had successfully demonstrated the feasibility of macular translocation to treat patients with subfoveal CNV secondary to AMD.

Ninomiya and associates ${ }^{26}$ later described a modification of Machemer's technique. They performed a complete vitrectomy followed by retinal detachment. During the first procedure, the detachment was created by infusing sodium hyaluronate into the subretinal space through a small retinotomy. However, this was found to be difficult and an exterior approach was used for the remaining cases. A $180^{\circ}$ retinal flap was created and elevated to expose the underlying tissue. After removing the neovascular membranes, the retina was repositioned and shifted, resulting in a retinal fold and also a strip of exposed RPE. Of the 3 cases, 2 patients had dramatic visual improvement, with 1 patient requiring a second operation to stabilise vision. The third patient's vision worsened significantly as a result of a retinal detachment.

In an effort to minimise the proliferative vitreoretinopathy (PVR) encountered by both Machemer and Ninomiya, we developed a technique by which the retina could be detached, the sclera shortened, and the retina reattached at a new location on the RPE without the creation of a large peripheral retinotomy. ${ }^{27,28}$ For this procedure, a balloon-like retinal detachment was formed by infusing balanced salt solution into the subretinal space through either a sclerotomy ${ }^{28}$ or a retinotomy. Once the retina was detached, a crescent of sclera one equatorial quadrant long and up to $4 \mathrm{~mm}$ wide was marked, dissected to three-quarters of total thickness, and excised. The bed edges were approximated and sutured together with 5-0 Dacron sutures. The detached retina was then flattened and the redundant retina migrated away from the chorioscleral infolding to facilitate macular translocation.

Following demonstration of this technique on rabbits, 3 eyes of 3 patients with recurrent subfoveal CNV and poor central vision were treated..$^{11}$ The same basic method as described above was used for all 3 eyes, although it is important to note that the retinal detachment was created via transretinal hydrodissection through several small punctate posterior retinotomies instead of trans-scleral hydrodissection.

Of the 3 treated eyes, 2 patients had significantly improved vision (20/160 to $20 / 30$ and $20 / 160$ to $20 / 70$ ) with only minor complications, whereas the other patient had a final visual acuity of 20/70 only after several follow-up surgeries to correct post-operative complications.

\section{Rationale}

Although the exact pathogenesis of CNV in eyes with AMD is not completely known, the idea of moving the fovea to a position overlying a relatively normal RPE/ choriocapillaris/Bruch's membrane complex is attractive. By moving the fovea away from the abnormal blood vessels it is possible to re-establish a more normal retinal subretinal physiology and the CNV can, then, be treated with laser photocoagulation without destroying the foveal centre.

This article describes our experience with limited macular translocation and our current surgical technique.

\section{Classification of macular translocation}

Currently, there are several different surgical techniques reflecting different strategies and surgical approaches to relocate the macula. Based on these various surgical techniques, we have created a classification that conforms to each surgical approach: ${ }^{29}$

1. Macular translocation with punctate or no retinotomy/ retinotomies: for those procedures that rely on small and self-sealing retinotomies or no retinotomy. Those procedures can be with (1a) or without (1b) chorioscleral shortening. Moreover, chorioscleral shortening can be achieved through chorioscleral infolding or chorioscleral outfolding.

2. Macular translocation with incomplete circumferential retinotomy: for those procedures with large but less than $360^{\circ}$ circumferential retinotomy.

3. Macular translocation with $360^{\circ}$ retinotomy: for those techniques using complete peripheral circumferential retinotomy.

Our current technique involves the use of small punctate self-sealing retinotomies to create a subtotal retinal detachment; relies on chorioscleral infolding to create retinal redundancy; and fluid-air exchange associated with post-operative upright positioning to achieve inferior translocation of the fovea (Fig. 1).

\section{Indications for limited macular translocation}

Currently, limited macular translocation (LMT) is being performed in patients with subfoveal CNV due to a variety of aetiologies including $\mathrm{AMD},{ }^{30}$ pathological myopia, ocular histoplasmosis syndrome (OHS), angioid streaks, idiopathic CNV and multifocal choroiditis. ${ }^{31}$ Because subfoveal $\mathrm{CNV}$ is more prevalent in patients with AMD, our experience is larger in these subset of patients.

Recently, a report of a case demonstrated the feasibility of using LMT for the management of eyes with central RPE defect following submacular surgery. ${ }^{32}$ We believe that the spectrum of conditions in which macular translocation is clinically useful will expand in the future.

Some relevant histopathological considerations of surgically excised choroidal neovascularisation

Surgically excised CNV associated with AMD from patients enrolled in the Submacular Surgery Trials showed that membranes were more often located beneath the RPE compared with non-AMD CNV. ${ }^{22}$ Furthermore, photoreceptors were present in 28 (approximately 46\%) of the 61 specimens from the AMD group. Since the location of the subfoveal $\mathrm{CNV}$ 


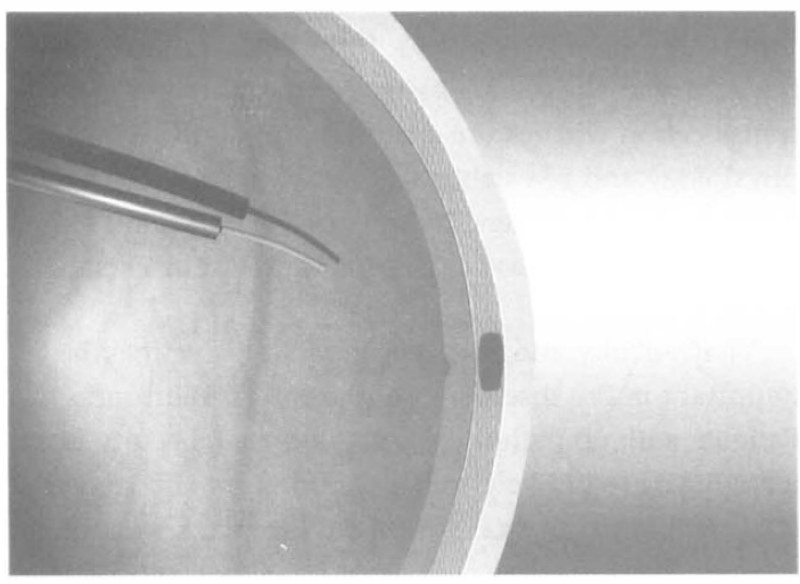

(a)

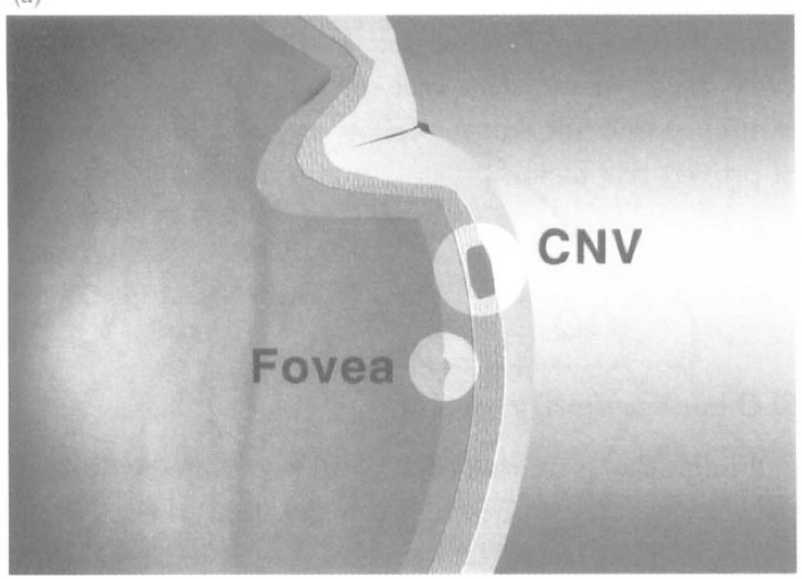

(c)

(subretinal vs sub-RPE) probably is associated with the visual prognosis after submacular membranectomy, those findings may explain the disappointing initial results of surgical removal of CNV secondary to AMD.

In a comprehensive histopathological evaluation of 760 eyes with AMD, Green and Enger ${ }^{33}$ showed that in disciform scars greater than $0.2 \mathrm{~mm}$ in thickness, only approximately $25 \%$ of the surface of the scar had some remaining photoreceptor cells. They observed greater photoreceptor atrophy as the disciform scars increased in size and thickness. These findings suggest that during early stages of the disease, when the lesions are still small and less fibrovascular scarring is present, there may be more viable photoreceptors and, hence, more potential vision to be rescued with the re-establishment of a normal subretinal space.

\section{Clinicopathological considerations}

One important aspect to be considered is the mechanism of vision loss in patients with subfoveal CNV secondary to AMD. All the evidence provided by anatomopathological and clinical studies of the natural history of eyes with AMD suggests a progressive anatomical and visual deterioration with time. The understanding of the mechanisms of vision loss is important to identify at which stage of the disease the neurosurgery retina is still

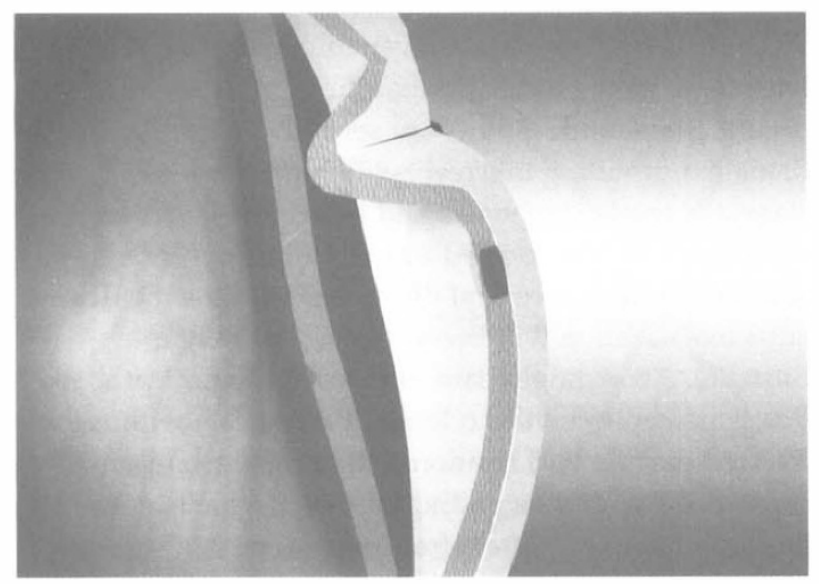

(b)

Fig. 1. Macular translocation procedure. (a) Detaching the retina by injecting balanced salt solution into the subretinal space using a subretinal infusion needle. (b) Shortening the sclera will create retinal redundancy. (c) The fovea is displaced away from the underlying choroidal neovascularisation (CNV) and laser treatment is administered to prevent further neovascular proliferation.

viable for a surgical intervention. No existing treatment will recover the function of a degenerated sensory retina that has no viable photoreceptors.

Early in the course of the disease process, the most common complaints of patients are metamorphopsia and central blurring. At this early stage, the visual acuity may be compromised and central fixation will become progressively unstable, but the patient will still retain foveal fixation. Initially, the anatomical changes responsible for the visual impairment are: (1) abnormal RPE function (retinol metabolism) and impaired nutrient/waste exchange across the RPE and Bruch's membrane; (2) relative retinal ischaemia/hypoxia secondary to an abnormal RPE-Bruch's membrane-choriocapillaris complex; (3) retinal oedema and subretinal fluid; and (4) retinal and subretinal haemorrhages. Most of these mechanisms can be reduced or reversed with a successful foveal translocation. With the re-establishment of a more normal subretinal space, it is possible to recover, at least partially, the normal subretinal physiology with subsequent visual recovery. The best candidates for macular translocation are therefore those with recent-onset metamorphopsia or disturbance in central vision due to new or recurrent $\mathrm{CNV}$, before the onset of more severe and permanent damage in the foveal retina.

Without treatment, the retina will continue to suffer from the injury caused by the CNV, and the photoreceptors will be progressively harmed until the 
visual function becomes irreversibly compromised. At late stages of the disease, when fibrovascular scarring occurs, the photoreceptors are severely and permanently damaged, resulting in irreversible functional loss. At this stage, the metamorphopsia and blurring will become less prominent and the central vision will be severely compromised by a central dense scotoma. As the disease advances, fixation will become progressively more unstable. Accordingly, late in the pathological process, visual perception will no longer be possible within the affected macula and fixation will become exclusively eccentric. The understanding of this mechanism is important because, after irreversible foveal photoreceptor loss, affected eyes are unlikely to achieve good functional recovery even after effective macular translocation.

\section{Pre-operative evaluation}

The patient selection criteria continue to evolve based on our experience with this surgical procedure. In general, patients with relatively early, small, well-defined subfoveal CNV who retain good pre-operative central retinal function are the best candidates.

\section{Retinal function testing}

The treatment window when the damage is still recoverable probably correlates with pre-operative neurosensory retinal function. Consequently, the measurement and quantification of macular function will provide critical information for patient selection and valuable prognostic clues for macular translocation. Currently, several new tests of macular function are available to the clinician. Although the central retinal function can be accessed by numerous tests such as bestcorrected visual acuity measurement, scanning laser ophthalmoscope (SLO) microperimetry, focal electroretinography, laser interference patterns, entoptic phenomena, contrast sensitivity and reading speed, the most widely used test for patient screening is the standard Snellen visual acuity. One important use of these supplements to standard Snellen acuity measures is to better predict post-operative visual acuity. Our experience has shown that SLO microperimetry is very helpful for the pre-operative evaluation and patient selection for macular translocation.

\section{Visual acuity}

Because visual acuity is a measure of retinal function, the better the pre-operative visual acuity, the better the retinal function, and hence, the higher the likelihood of achieving good vision after effective macular translocation.

In our series, good pre-operative best-corrected visual acuity was significantly correlated with better visual outcome after LMT. ${ }^{30}$ Although good pre-operative visual acuity is indicative of relatively good potential macular function, poor visual acuity does not necessarily mean irreversible vision loss. If the retinal damage has not achieved an irreversible status, a successful macular translocation will restore a more normal retinal physiology and re-establish, at least partially, the visual function. In fact patients with poor visual acuity $(\leqslant 20 / 320)$ experienced the largest amount of visual improvement.

Visual acuity and status of the fellow eye may be important in the discussion of the risk/benefit ratio. Patients with no pathology and good visual acuity in the fellow eye are unlikely to have immediate change in the quality of life even after successful macular translocation surgery. The benefit is noticeable when we consider that the natural history of fellow eyes in patients with $\mathrm{CNV}$ secondary to AMD is associated with significant risk of CNV development. This risk can be estimated with careful examination of the fellow eye and identification of proven risk factors.

\section{SLO microperimetry}

Although the exact role of SLO microperimetry during patient selection for macular translocation in eyes with subfoveal CNV secondary to AMD is yet to be established, this test appears to be helpful in the preoperative evaluation of the central retinal function and in the selection of ideal candidates for the translocation procedure.

One of the most important abilities of this test is to disclose the fixation pattern, which reflects primarily the stability of fixation and the preferred fixation location. As the central retinal function deteriorates, the area of fixation enlarges, proportional to the inability to maintain stable fixation in the centre of the FAZ. In the late stages of the disease, the eye will no longer fixate within the FAZ centre and fixation will be exclusively eccentric. The complete loss of central fixation probably reflects permanent impairment of the central neurosensory retina, and therefore indicates a poor candidate for macular translocation.

SLO microperimetry can also measure the sensitivity within the central retina. Although the retinal sensitivity correlates with the amount of retinal damage present pre-operatively, we have seen cases in which areas of dense scotoma recovered partial retinal function after effective macular translocation. This suggests that even when microperimetry indicates the presence of dense scotoma areas, in cases that still present some central fixation there is still a treatment window that allows for some functional recovery in those otherwise permanently damaged retinal areas. However, once fixation becomes exclusively eccentric, it is unlikely that it will become central even after an effective macular translocation. 

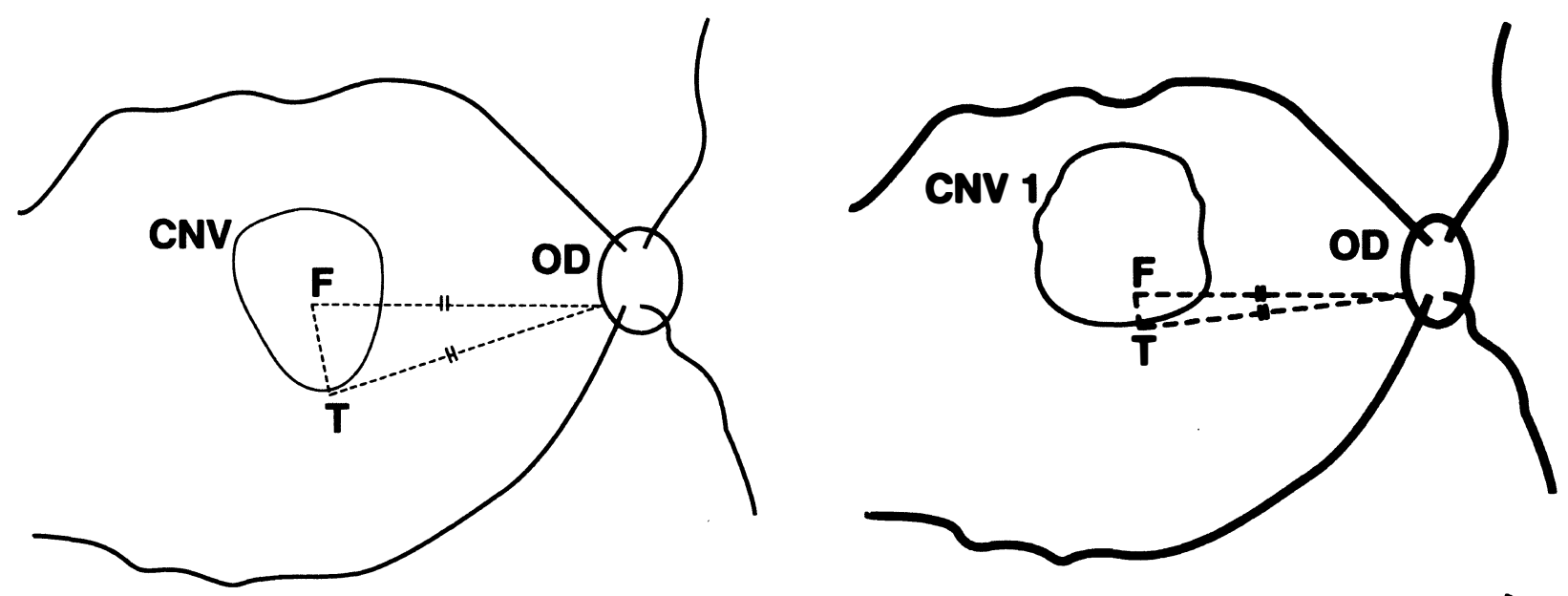

(a)

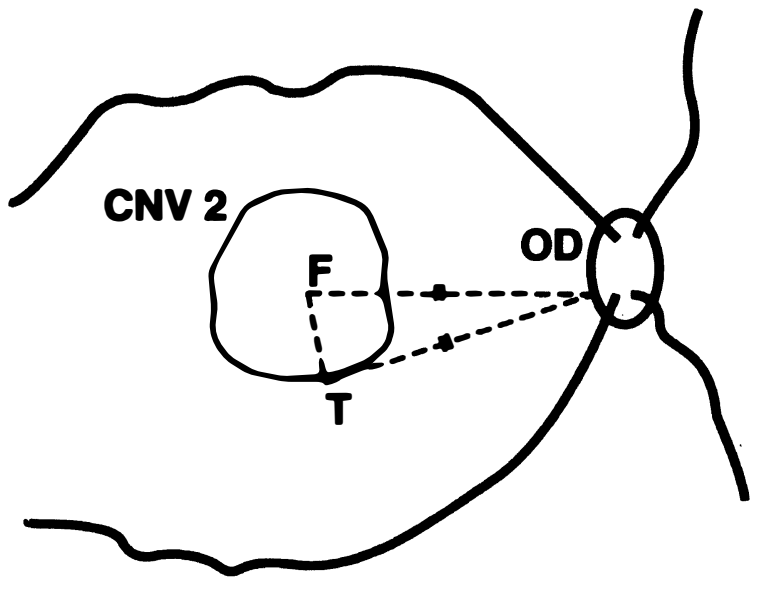

(c)
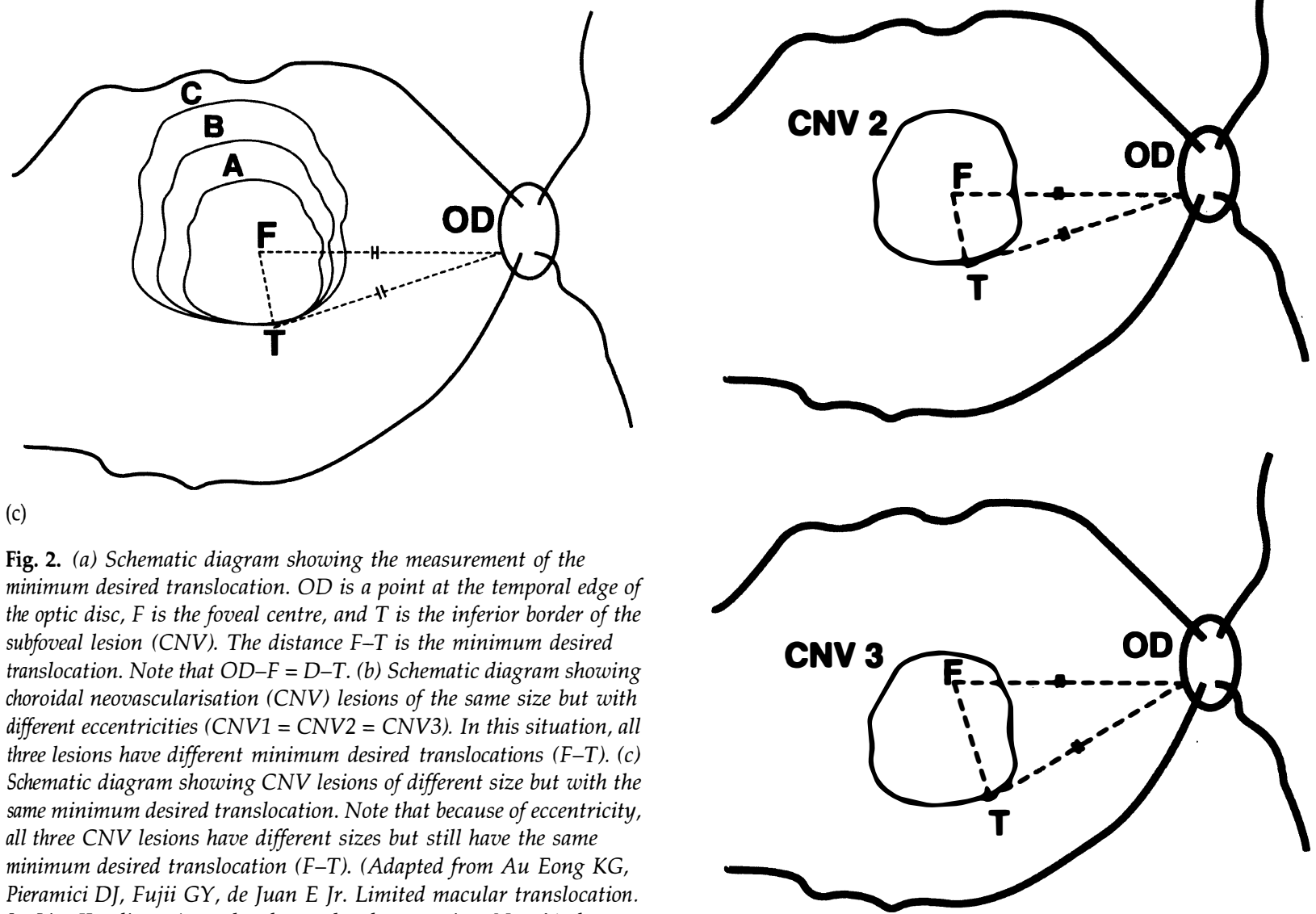

Fig. 2. (a) Schematic diagram showing the measurement of the minimum desired translocation. $O D$ is a point at the temporal edge of the optic disc, $F$ is the foveal centre, and $T$ is the inferior border of the subfoveal lesion $(C N V)$. The distance $F-T$ is the minimum desired translocation. Note that $O D-F=D-T$. (b) Schematic diagram showing choroidal neovascularisation (CNV) lesions of the same size but with different eccentricities $(C N V 1=C N V 2=C N V 3)$. In this situation, all three lesions have different minimum desired translocations $(F-T)$. (c) Schematic diagram showing CNV lesions of different size but with the same minimum desired translocation. Note that because of eccentricity, all three $C N V$ lesions have different sizes but still have the same minimum desired translocation ( $F-T$ ). (Adapted from Au Eong $K G$, Pieramici DJ, Fujii GY, de Juan E Jr. Limited macular translocation. In: Lim IJ, editor. Age-related macular degeneration. New York: Marcel Dekker, in press.)

\section{Fluorescein angiography}

A good quality pre-operative fluorescein angiogram is required, obtained preferably within 1 week of the surgery. Using the pre-operative angiogram, the lesion's characteristics can be evaluated and the minimum desired translocation measured.

\section{Minimum desired translocation}

One important anatomical consideration is the distance between the FAZ center and the inferior border of the $\mathrm{CNV}$ complex, when inferior translocation is planned. This distance corresponds to the minimum amount of foveal displacement required to have the fovea moved

(b)

just away from the CNV complex. This distance is defined as the minimum desired translocation (Fig. 2). Ideally, there should be more foveal displacement than the minimum desired translocation in order to preserve more central neurosensory retina. With a larger distance between the fovea and the CNV complex, the scotoma created by the laser photocoagulation will have less effect in the central vision. On the other hand, it is possible that by achieving a larger foveal displacement, a postoperative diplopia may become more symptomatic.

After measuring the minimum desired translocation the chances of achieving effective macular translocation are estimated using the knowledge of the amount of foveal displacement obtained in previous cases. 
Another important consideration is the analysis of the characteristics of the CNV lesion, because they may have major implications in the natural history of the disease. Classic subfoveal CNV lesions secondary to AMD are associated with rapid vision deterioration, ${ }^{16}$ while occult patterns are associated with less aggressive vision loss. ${ }^{34}$ This differentiation will be important in the discussion of the risk/benefits ratio, as one patient may elect not take the risks of the surgery immediately if the natural history of a specific case is stable visual acuity for some period of time.

\section{Current surgical technique for LMT}

LMT has been performed on more than 450 patients at the Wilmer Ophthalmological Institute over the past 4 years. Our translocation technique is still evolving as we gain more surgical experience and have an ever-growing pool of post-operative patients to follow. The technique described here reflects our most recent limited retinal translocation procedure.

The pre-operative set-up includes standard three-port pars plana vitrectomy equipment and several devices that were developed specifically for this procedure.

In our practice, before beginning surgery, an intraocular humidifier, infusion needle and retinal manipulator are set up for use. The intraocular humidifer (MoistAir humidifying chamber, RetinaLabs.com Atlanta, GA) resides in-line between the three-way stopcock and the infusion cannula. Humidified air is used during fluid-air exchange in phakic eyes to keep the lens clear. Experiments in rabbits have shown that use of humidified air instead of room air increases the time to gas-induced feathering of the lens by $80 \% .{ }^{35}$ In addition, there is increasing evidence that humidified air may prevent retinal damage.

Still in the pre-operative set-up, a retinal hydrodissection cannula is connected to the syringe of a viscous fluid injector, which should be filled with a balanced salt solution and the high-pressure airline cap placed on the rear of the syringe. The viscous fluid injector pressure should be set so that approximately 4 drops per second form on the end of the needle. The adjustable and constant pressure created by the viscous fluid injector allows for a more controlled detachment. During the procedure, the retina is intentionally detached by injecting fluid into the subretinal space through several small punctate retinotomies using the 41 gauge retinal hydrodissection cannula (MADLAB retinal hydrodissection cannula, Bausch \& Lomb Surgical, St Louis, MO).

It is often desirable to grasp and gently manipulate the retina during fluid-air exchange. Initially this was done using a soft-tipped cannula. We found this not to be ideal and instead we have designed a retinal manipulator (Bausch \& Lomb Surgical, St Louis, MO) specifically for this purpose. The manipulator is a blunt 20 gauge cannula with three small holes in its tip. The holes in the manipulator are small enough to allow the retina to be grasped with minimal damage using aspiration but still

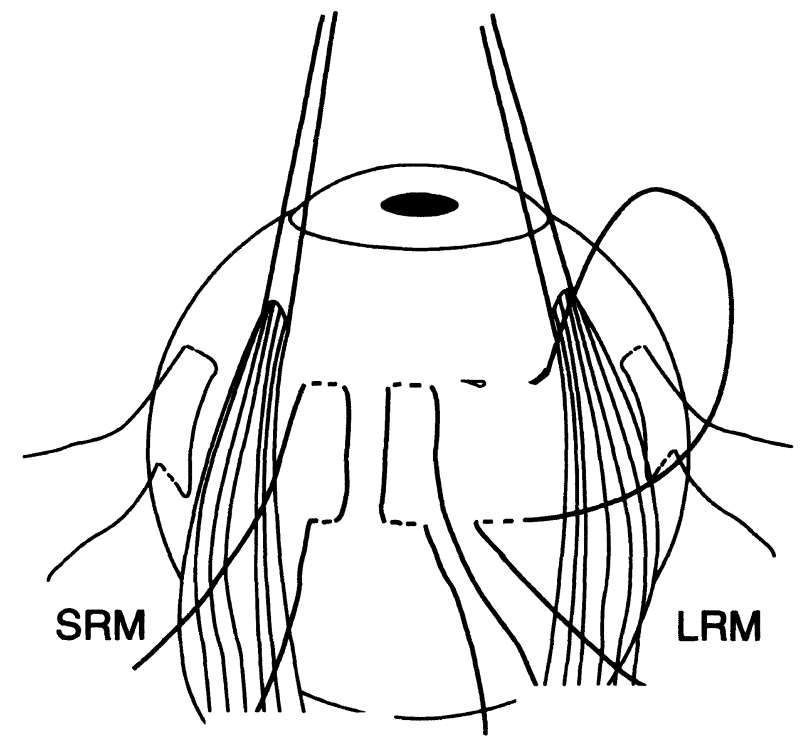

Fig. 3. Scleral sutures are pre-placed in horizontal mattress fashion before retinal detachment. The sutures are held with serrefines until later in the procedure when they are tightly knotted to shorten the sclera. SRM, superior rectus muscle; LRM, lateral rectus muscle. (Adapted from Au Eong KG, Pieramici DJ, Fujii GY, de Juan E Jr. Limited macular translocation. In: Lim IJ, editor. Age-related macular degeneration. New York: Marcel Dekker, in press.)

large enough for efficient fluid-air exchange. The manipulator is connected to a reflux handle to provide fine control over the grasping and release of retinal tissue. The combination of the reflux handle with the small holes in the tip of the retinal manipulator prevents the retina from being incarcerated.

In some cases, typically in those eyes that have received previous laser photocoagulation, we have found the macula to be abnormally adherent to the underlying RPE. In these eyes, subdissection with a pick through an access retinotomy is required to achieve complete macular detachment. We have developed a new extendable flexible pick (Bausch \& Lomb Surgical, St Louis, MO) to facilitate this manoeuvre. ${ }^{36}$

During the procedure, we routinely administer our patients intravenous corticosteroids to decrease the incidence of PVR.

Initially during the surgery, a $270^{\circ}$ peritomy is performed and two rectus muscles are isolated and retracted on 2-0 silk sutures. The superior and temporal rectus muscles are used for an inferior macular translocation and the temporal and inferior muscles are isolated for a superior macular translocation.

In order to obtain scleral imbrication, five 5-0 Dacron imbrication sutures (Sherwood, Davis, \& Geek, Wayne, $\mathrm{NJ}$ ) are preplaced in a horizontal mattress fashion before detaching the retina and then tightening once the retinal detachment is complete (Fig. 3). This creates a chorioscleral infolding and decreases the circumference of the globe, resulting in scleral shortening and redundant retina.

The location of the sutures depends on the direction of desired translocation. If inferior translocation is desired, one suture is placed nasal to the superior rectus muscle, three sutures are placed between the superior and lateral 


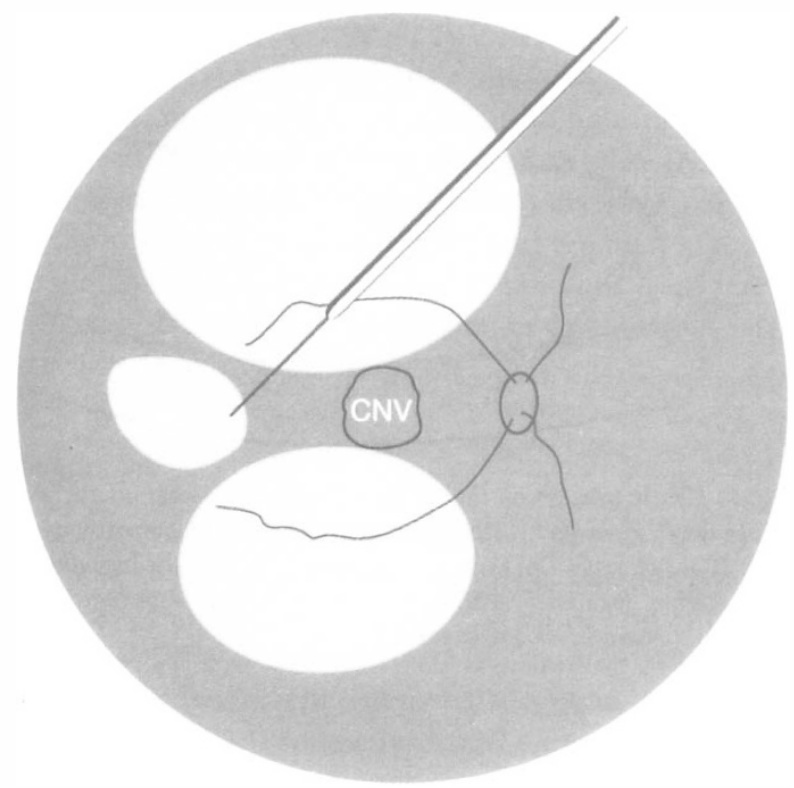

Fig. 4. Retinomies should be created near the superotemporal vascular arcades, inferotemporal vascular arcade and temporal to the macula, in that order. As few retinotomies as possible should be created, with three sites generally being sufficient. (Adapted from Au Eong KG, Pieramici DJ, Fujii GY, de Juan E Jr. Limited macular translocation. In: Lim IJ, editor. Age-related macular degeneration. New York: Marcel Dekker, in press.)

rectus muscles, and one suture is placed inferior to the lateral rectus muscle. For a superior translocation, the inferotemporal sclera/choroid is shortened by placing one suture superior to the lateral rectus muscle, one suture nasal to the inferior rectus muscle, and three sutures within the inferotemporal quadrant. All sutures are placed in a mattress fashion with the passes running parallel to the limbus. The anterior bite is placed 3-4 mm posterior to the muscle insertions. The posterior bite is placed $6 \mathrm{~mm}$ behind the anterior bite. After placement, the sutures are left untied and clamped with serrefines

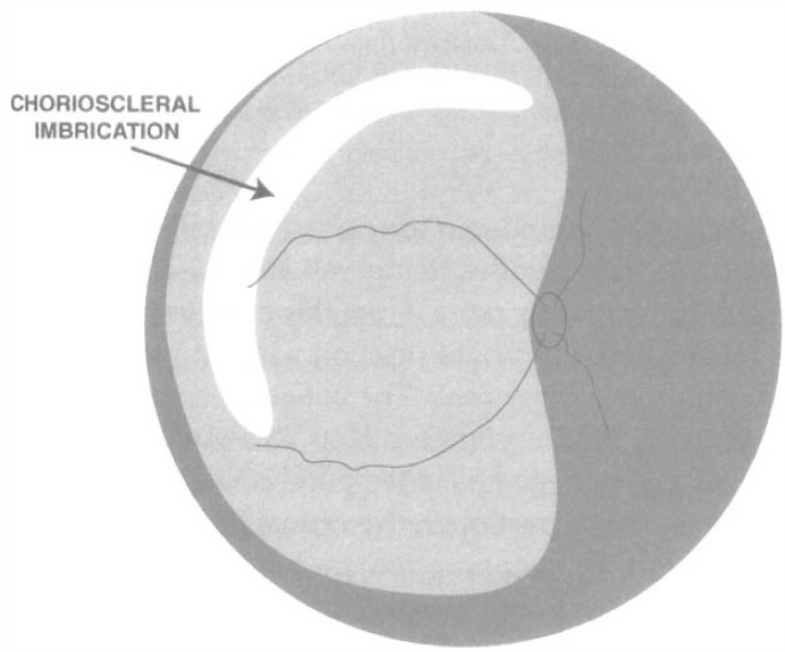

Fig. 5. Before tightening the imbricating sutures, the surgeon should confirm the complete detachment of the temporal retina, especially the macula. Note that the planned retinal detachment extends anteriorly beyond the area of chorioscleral infolding. (Adapted from Au Eong KG, Pieramici DJ, Fujii GY, de Juan E Jr. Limited macular translocation. In: Lim IJ, editor. Age-related macular degeneration. New York: Marcel Dekker, in press.) until later in the procedure. Although this technique creates a chorioscleral unfolding with theoretically minimal radial retinal reduncancy, the circumferential shortening of the eyeball created with this technique creates significant shortening in the vertical axis of the eyewall at the level of the fovea with consequent creation of a significant amount of vertical retinal redundancy at the fovea.

Following suture placement, a standard three-port pars plana vitrectomy is performed using a trocar-microcannula system that protects the delicate 41 gauge hydrodissection infusion cannula during insertion through the sclerotomy. During the vitrectomy, care should be taken to ensure the posterior hyaloid detachment.

The 41 gauge retinal hydrodissection cannula is then introduced through a trocar system and used to create detachment of the temporal retina through self-sealing retinotomies. As few retinotomies as possible (usually three, if possible) should be created, beginning with two sites in the superotemporal and inferotemporal regions, with the final site being just temporal to the macula (Fig. 4). At each site, the viscous fluid injector is used to inject saline solution into the subretinal space, causing a focal detachment of the retina. Each infusion continues until the focal detachment extends to the far periphery of the temporal retina. Importantly, the detachment of the temporal retina should extend peripherally beyond the imbricating sutures (Fig. 5).

Following infusion of saline at all selected sites, the focal detachments will often not be connected. A humidified fluid-air exchange is then performed to push the subretinal fluid posteriorly, connecting the blisters and detaching the macula and temporal retina all the way to the optic disc (Fig. 6). It is advisable to wait patiently until no more hydrodissection is under way. In this case, the air is exchanged back to fluid and the retinal manipulator can be used to gently manipulate the retina under low suction to grasp part of the detached retina. Gentle traction of the detached retina can be performed to ensure that the posterior retina is free and fully detached (Fig. 7). This step is very important because it may determine the success of the surgery. If the entire macula is not detached up to the temporal edge of the optic disc, the retina will not be able to rotate around the axis of the optic nerve. The air is then removed and the retina can be checked once more to confirm that it is fully detached, especially in the macular region. If those manoeuvres are not effective in fully detaching the macula after several attempts, the use of a subretinal pick is indicated to break abnormal adhesions (Fig. 8). ${ }^{36,37}$

It is important not to detach the nasal retina during this procedure. We have found that avoiding the nasal retinal detachment minimises the chance of a postoperative retinal fold being formed at the fovea.

Once the macula is completely detached, the air infusion into the eye is then changed back to fluid. By stopping the infusion and temporarily removing one of the trocar plugs the intraocular pressure is lowered. 


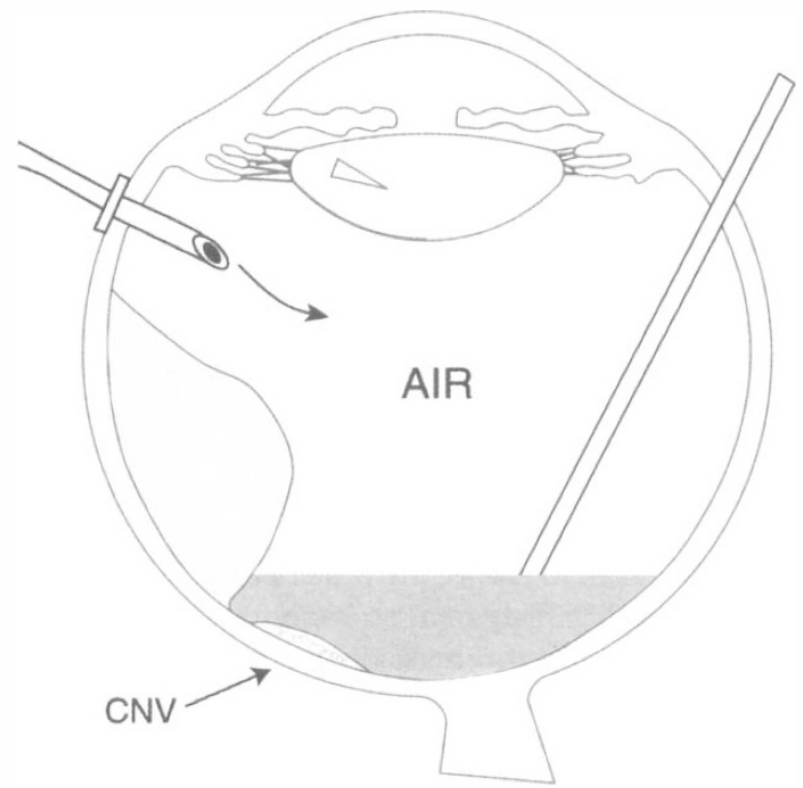

Fig. 6. After a complete fluid-air exchange, the air forces the subretinal fluid to hydrodissect the macula off the diseased retinal pigment epithelium and $C N V$ complex. (Adapted from Au Eong $K G$, Pieramici DJ, Fujii GY, de Juan E Jr. Limited macular translocation. In: Lim IJ, editor. Age-related macular degeneration. New York: Marcel Dekker, in press.)

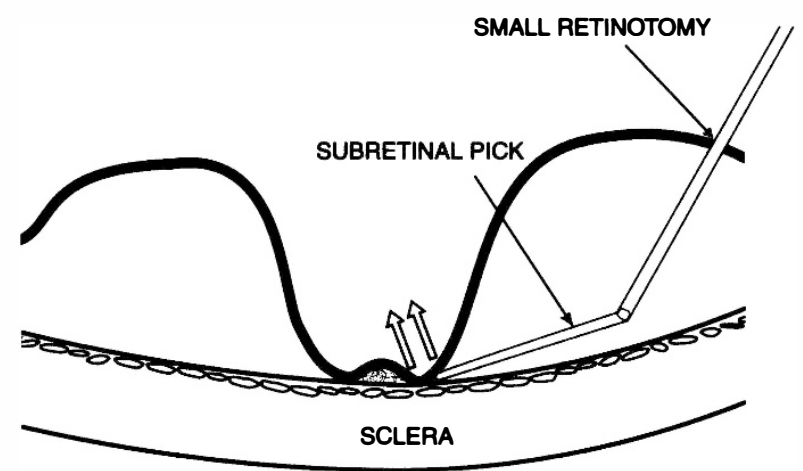

Fig. 8. Abnormal adhesion between the neurosensory macula and the underlying lesion, usually in laser scar regions, may require the use of a subretinal pick for subretinal blunt dissection. (Adapted from $\mathrm{Au}$ Eong KG, Pieramici DJ, Fujii GY, de Juan E Jr. Limited macular translocation. In: Lim IJ, editor. Age-related macular degeneration. New York: Marcel Dekker, in press.)

Then, the preplaced scleral sutures are pulled up, tied and cut. Note that tightening the sutures should be performed under fluid and the retina should be detached all over the chorioscleral infolding. Still under fluid, the peripheral retina should be inspected to rule out any inadvertent retinal breaks.

If an inferior translocation is needed, following tightening of the imbrication sutures a $75-90 \%$ fluid-air exchange is performed. For a superior translocation, a retinotomy is created through which subretinal fluid is evacuated during fluid-air exchange with gentle upward retinal manipulation.

The surgical procedure will finish with closure of the sclerotomies and conjunctival incision. Subconjunctival antibiotic and corticosteroid are then injected, a combination antibiotic-steroid ointment is applied and the eye is patched and covered with an eye shield.

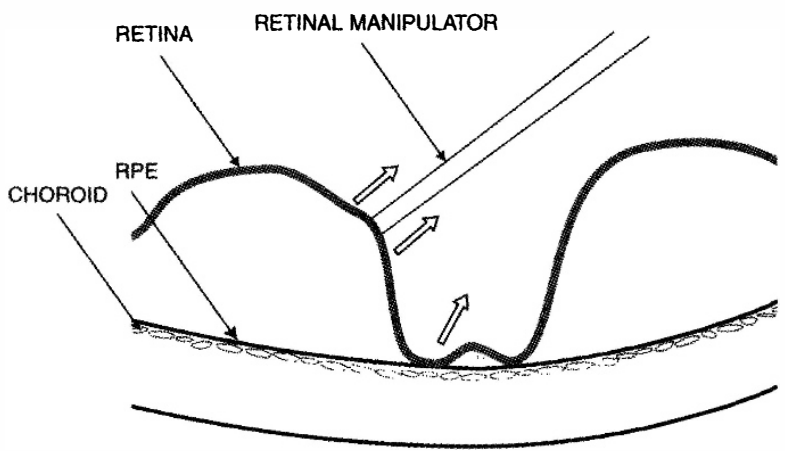

Fig. 7. Gentle traction on the retina with a retinal manipulator encourages the complete detachment of the macula. (Adapted from Au Eong KG, Pieramici DJ, Fujii GY, de Juan E Jr. Limited macular translocation. In: Lim IJ, editor. Age-related macular degeneration. New York: Marcel Dekker, in press.)

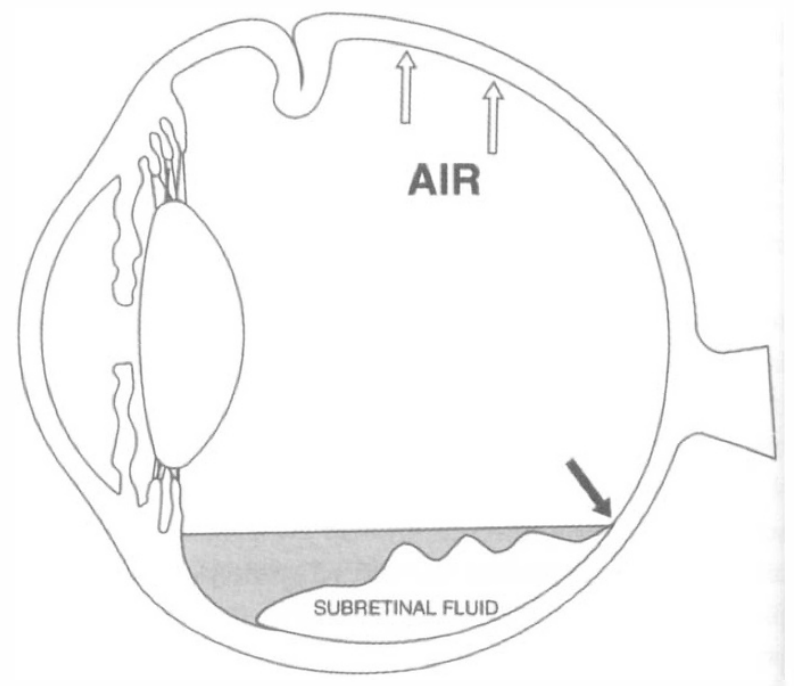

Fig. 9. With the patient in an upright position post-operatively, the air buoyancy supports the superior retina while the subretinal fluid is accumulated inferiorly, dragging the fovea downwards relative to the underlying CNV lesion. (Adapted from Au Eong KG, Pieramici DJ, Fujii GY, de Juan E Jr. Limited macular translocation. In: Lim IJ, editor. Age-related macular degeneration. New York: Marcel Dekker, in press.)

Patients who undergo inferior translocation are turned on the operative (temporal) side for about $5 \mathrm{~min}$, still in the operating room. From this position, the patient is moved into an upright position without turning the patient on his or her back. The patient is then instructed to keep the head upright for $24 \mathrm{~h}$. This procedure will allow for a more complete temporal retinal detachment and will avoid a post-operative retinal fold being formed at the fovea. With appropriate post-operative positioning, the gravitational force of the subretinal fluid and the flotation force of the intravitreal air bubble will be aligned to effect inferior macular translocation (Fig. 9).

When intraocular air no longer precludes the view of the macula, usually between 3 and 7 days postoperatively, a fluorescein angiogram should be taken to identify and guide the treatment of the CNV. An effective macular translocation will move the fovea away 
from the area of $\mathrm{CNV}$. In this case, the neovascularisation is no longer subfoveal and laser photocoagulation treatment can be used as an attempt to halt further proliferation of the blood vessels and stabilise the degenerative process. The patients are re-checked in 3 weeks to rule out persistent $\mathrm{CNV}$ and are then followed up with serial examinations every 3 months or sooner if they complain of decreasing vision or any retinal detachment symptoms.

\section{Results}

Recently, we have reported our initial experience and the outcome of 102 consecutive eyes of 101 patients who underwent inferior limited macular translocation for subfoveal CNV secondary to $\mathrm{AMD}^{30}$ and 23 consecutive eyes of 22 patients with subfoveal $\mathrm{CNV}$ due to causes other than AMD. ${ }^{31}$ An analysis of the complications was reported in a separate manuscript. ${ }^{38}$

\section{AMD}

A total of 102 eyes of 101 patients with subfoveal CNV associated with AMD are included in our initial report. ${ }^{30}$ Only cases of inferior LMT without scleral resection were included. The translocation with scleral resection and superior translocation are technically quite different and were not included in this series. The baseline characteristics were as follows. The ages of the patients treated ranged from 41 to 89 years (median 76 years); there were 59 men and 42 women. The range of preoperative Snellen visual acuity was $20 / 40$ to counting fingers (median 20/160) and had the following distribution: 20 patients with pre-operative visual acuity between $20 / 40$ and 20/80, 41 patients between 20/100 and 20/160, 25 patients between 20/200 and 20/320, 7 patients between $20 / 400$ and 20/640, 7 patients $20 / 800$ or worse. Two patients did not have their pre-operative visual acuity recorded. Nearly $60 \%$ of the study group had a pre-operative visual acuity better than $20 / 200$. The lesion size ranged from less than 1 Macular Photocoagulation Study (MPS) disc area (DA) to 9 DAs (median 3 DAs). In this initial series, more than one-third of the lesions were greater than 3.5 MPS DAs in size prior to translocation.

In $62 \%$ of cases effective macular translocation was achieved. With inferior LMT, the amount of macular translocation achieved ranged from 200 to $2800 \mu \mathrm{m}$ (median $1200 \mu \mathrm{m}$ ). More specifically, 75\% of patients experienced at least $950 \mu \mathrm{m}$ of retinal movement and $25 \%$ experienced movement of $1500 \mu \mathrm{m}$ or more.

By 3 months and 6 months post-operatively, 37\% and $48 \%$ of patients respectively experienced an improvement in visual acuity of 2 Snellen lines or more from their pre-operative measurement, and $31 \%$ and $49 \%$ of the eyes respectively achieved a visual acuity better than 20/100. Sixteen per cent of the eyes experienced 6 or more Snellen lines of visual improvement by 6 months of follow-up. At 3 and 6 months post-operatively, $41 \%$ and $32 \%$ respectively remained within 1 line of visual acuity and $23 \%$ and $29 \%$ respectively lost 2 or more lines of visual acuity. Ten per cent of the eyes experienced 6 or more Snellen lines of visual loss.

To identify predictive factors association with change from baseline visual acuity at 3 and 6 months multiple regression models were used. Significant predictive factors included good pre-operative visual acuity, achieving the desired amount of post-operative foveal displacement, a greater amount of post-operative foveal displacement and recurrent $\mathrm{CNV}$ at baseline.

The size of the lesion at presentation measured in MPS DAs was not predictive of visual acuity, probably due to eccentricity of lesions related to the fovea. We did not consider the eccentricity of the $\mathrm{CNV}$ lesions during measurements and thus a large lesion centred superior to the fovea might require only a small amount of translocation so that the inferior portion was no longer under the foveal centre. The critical measurement is the minimum desired translocation.

Twenty-two of the 102 eyes in the study group had a history of previous laser treatment and thus presented with a subfoveal recurrence. While the initial visual acuities did not differ significantly, the visual acuities were significantly better at 3 and 6 months in eyes with recurrent $\mathrm{CNV}$ as compared with eyes with new subfoveal CNV. The reason is probably that these patients represent a select group of individuals who, having undergone previous laser treatment, are better educated, and more aware of the necessity to see their ophthalmologist as soon as possible when a visual change occurs, and thus present earlier, when the central neurosensory retina is still viable.

Not surprisingly, good pre-operative visual acuity was one of the most significant predictors of short-term visual outcome in this study. This reflects the fact that visual acuity is a functional test of the macular retinal cells, and good presenting vision indicates good potential retinal cell function. However, it is important to note that some patients with poor visual acuity $(\leqslant 20 / 320)$ experienced a large amount of visual improvement in our series, suggesting that additional tests of baseline retinal cell viability, such as SLO microperimetry, are indicated for optimum patient selection.

\section{Complications}

In order to study the complications associated with limited macular translocation we have retrospectively reviewed the initial $153^{\circ}$ consecutive cases of LMT surgery used to treat subfoveal choroidal neovascularisation. ${ }^{38}$ We have included in this study early cases on which we performed LMT in order to identify factors correlated with the development of an ocular complication and to investigate the existence of a surgical procedure learning process.

At least one complication occurred in 52 eyes (35\% of 153 eyes). Of 153 eyes, the intraoperative complications identified included macular hole $(n=12)$, retinal break $(n=8)$, subretinal haemorrhage $(n=7)$, vitreous haemorrhage $(n=3)$, scleral perforation $(n=3)$, and 
choroidal haemorrhage $(n=1)$. The post-operative complications identified included retinal detachment $(n=25)$, retinal breaks $(n=13)$, macular fold $(n=7)$, vitreous haemorrhage $(n=4), \mathrm{CNV}$ at the retinotomy site $(n=2)$, choroidal haemorrhage $(n=1)$ and suspected endophthalmitis $(n=1)$. Eyes developing retinal detachment, subretinal haemorrhage and macular fold had significantly more loss of vision than eyes without each of these complications $(p<0.05)$. The presence of predominantly classic $\mathrm{CNV}$, the occurrence of an intraoperative retinal break, any intraocular haemorrhage, or macular fold formation were significantly associated with retinal detachment $(p<0.03)$. The incidence of any complication, retinal detachment and haemorrhage significantly decreased during the study period, suggesting a learning process $(p<0.03)$.

The most common and serious post-operative complication was retinal detachment. However, retinal detachment occurred more frequently early in the developing stages of the technique, and its incidence decreased significantly during the study period. In 18 of 25 cases of post-operative unplanned retinal detachment there was associated PVR. Four of the 25 eyes underwent successful detachment repair with pneumatic retinopexy using sulphur hexafluoride in the office. The remaining 21 eyes required repeat operations in the operating room for successful retinal reattachment. Currently, our incidence of retinal detachment following LMT is approximately $5-10 \%$.

Although many of the patients initially experienced torsional diplopia, these symptoms seem to lessen with time. No patient in our series required extraocular muscle surgery for diplopia correction. When treatment was required, prismatic correction appeared to be satisfactory.

\section{Persistent and recurrent CNV following LMT}

Complications have been reported to occur up to 3 months after LMT. After 3 months post-operative followup, the most common cause of vision loss is recurrence of CNV. Although we do not know either the exact incidence of persistence of recurrence of CNV after LMT, or the impact these occurrences have on visual acuity, a preliminary study has demonstrated that patients are still at significant risk of CNV recurrence after LMT. ${ }^{39}$ In the group of patients with effective macular translocation, the entire $\mathrm{CNV}$ complex was treated with laser photocoagulation, sparing the sensory fovea. The incidence of recurrence in this group was 35\% at 9 months. When recurrence happened, it occurred with equal frequency on the foveal side of the laser scar $(5 / 10)$ and on the non-foveal side of the laser scar $(5 / 10)$. Four of the five foveal-side recurrences were subfoveal, and accounted for the vision loss in foveal-side recurrence.

If the CNV complex persists subfoveally after LMT (non-effective macular translocation), a variety of treatment options are available including observation, laser ablation of the fovea, surgical resection of the $\mathrm{CNV}$ or photodynamic therapy. We tend not to perform repeat macular translocation for persistent or recurrent subfoveal CNV because initial experience resulted in increased incidence of retinal detachment and PVR.

\section{Summary}

The macular translocation procedure is a promising treatment modality as it offers patients a chance of potentially improving their vision to an acceptable level for reading and driving. Functionally, those patients with subfoveal CNV of recent onset without permanent foveal sensory retina damage have the greatest likelihood of successful macular translocation. Anatomically, the best candidates have a small minimum desired translocation, with healthy RPE/Bruch's membrane/choriocapillaris outside the lesion. The aetiology is usually AMD, but pathological myopia, ocular histoplasmosis syndrome, angioid streaks, idiopathic and multifocal-choroiditisrelated $\mathrm{CNV}$ might be considered. A variety of ocular complications can occur during or following LMT and some are associated with reduced post-operative visual acuity. Improved surgical techniques and experience may significantly reduce the incidence of these complications. The most important complication is retinal detachment, which can usually be repaired, although it may affect the final visual result.

The exact role of translocation is not yet established and a pilot multicentre randomised clinical trial is under way to compare the results of LMT with either photodynamic therapy or observation in eyes with subfoveal CNV secondary to AMD.

Supported in part by grants from the Foundation Fighting Blindness, the Macula Vision Research Foundation, and numerous private donors. G.Y.F. was supported by grants from the Foundation Fighting Blindness, the Macula Vision Research Foundation and The Wilmer Macular Research Foundation.

\section{References}

1. Ferris FL III, Fine SL, Hyman L. Age-related macular degeneration and blindness due to neovascular maculopathy. Arch Ophthalmol 1984;102:1640-2.

2. Bressler SB, Bressler NM, Fine SL, Hillis A, Murphy RP, Olk RJ, et al. Natural course of choroidal neovascular membranes within the foveal avascular zone in senile macular degeneration. Am J Ophthalmol 1982;93:157-63.

3. Bressler NM, Bressler SB, West SK, Fine SL, Taylor HR. The grading and prevalence of macular degeneration in Chesapeake Bay watermen. Arch Ophthalmol 1989;107:847-52.

4. Klein R, Klein BE, Jensen SC, Meuer SM. The five-year incidence and progression of age-related maculopathy: the Beaver Dam Eye Study. Ophthalmology 1997;104:7-21.

5. Klein R, Klein BE, Linton KL. Prevalence of age-related maculopathy: the Beaver Dam Eye Study. Ophthalmology 1992;99:933-43.

6. Vingerling JR, Klaver CC, Hofman A, de Jong PT. Epidemiology of age-related maculopathy. Epidemiol Rev 1995;17:347-60. 
7. Macular Photocoagulation Study Group. Risk factors for choroidal neovascularization in the second eye of patients with juxtafoveal or subfoveal choroidal neovascularization secondary to age-related macular degeneration. Arch Ophthalmol 1997;115:741-7.

8. Hirvela H, Luukinen H, Laara E, et al. Risk factors of agerelated maculopathy in a population 70 years of age or older. Ophthalmology 1996;103:871-7.

9. Klein R, Klein BE, Jensen SC, Cruickshanks KJ. The relationship of ocular factors to the incidence and progression of age-related maculopathy. Arch Ophthalmol 1998;116:506-13.

10. Treatment of Age-related Macular Degeneration with Photodynamic Therapy (TAP) Study Group. Photodynamic therapy of subfoveal choroidal neovascularization in agerelated macular degeneration with verteporfin: one-year results of 2 randomized clinical trials: TAP report. Arch Ophthalmol 1999;117:1329-45.

11. de Juan E Jr, Loewenstein A, Bressler NM, Alexander J. Translocation of the retina for management of subfoveal choroidal neovascularization. II. A preliminary report in humans. Am J Ophthalmol 1998;125:635-46.

12. Seo MS, Kwak N, Ozaki H, Yamada H, Okamoto N, Yamada $\mathrm{E}$, et al. Dramatic inhibition of retinal and choroidal neovascularization by oral administration of a kinase inhibitor. Am J Pathol 1999;154:1743-53.

13. Char DH, Irvine AI, Posner MD, Quivey J, Phillips TL, Kroll $\mathrm{S}$. Randomized trial of radiation for age-related macular degeneration. Am J Ophthalmol 1999;127:574-8.

14. Bressler NM. Submacular surgery. Are randomized trials necessary? Arch Ophthalmol 1995;113:1557-60.

15. Macular Photocoagulation Study Group. Laser photocoagulation of subfoveal neovascular lesions in agerelated macular degeneration: results of a randomized clinical trial. Arch Ophthalmol 1991;109:1220-31.

16. Macular Photocoagulation Study Group. Laser photocoagulation of subfoveal neovascular lesions of agerelated macular degeneration: updated findings from two clinical trials. Arch Ophthalmol 1993;111:1200-9.

17. Macular Photocoagulation Study Group. Persistent and recurrent neovascularization after laser photocoagulation for subfoveal choroidal neovascularization of age-related macular degeneration. Arch Ophthalmol 1994;112:489-99.

18. de Juan E Jr, Machemer R. Vitreous surgery for hemorrhagic and fibrous complications of age-related macular degeneration. Am J Ophthalmol 1988;105:25-9.

19. Blinder KJ, Peyman GA, Paris CL, Gremillion CM Jr. Submacular scar excision in age-related macular degeneration. Int Ophthalmol 1991;15:215-22.

20. Thomas MA. The use of vitreoretinal surgical techniques in subfoveal choroidal neovascularization. Curr Opin Ophthalmol 1992;3:349-56.

21. Thomas MA, Dickinson JD, Melberg NS, Ibanez HE, Dhaliwal RS. Visual results after surgical removal of subfoveal choroidal neovascular membranes. Ophthalmology 1994;101:1384-96.

22. Grossniklaus HE, Green WR. Histopathologic and ultrastructural findings of surgically excised choroidal neovascularization. Submacular Surgery Trials Research Group. Arch Ophthalmol 1998;116:745-9.

23. Lindsey P, Finkelstein D, D'Anna S. Experimental retinal rotation [abstract]. Invest Ophthalmol Vis Sci 1983;24(ARVO Suppl):242.
24. Machemer R, Steinhorst UH. Retinal separation, retinotomy, and macular relocation: II. A surgical approach for agerelated macular degeneration? Graefes Arch Clin Exp Ophthalmol 1993;231:635-41.

25. Machemer R, Steinhorst UH. Retinal separation, retinotomy, and macular relocation. I. Experimental studies in the rabbit eye. Graefes Arch Clin Exp Ophthalmol 1993;231:629-34.

26. Ninomiya $Y$, Lewis JM, Hasegawa T, Tano Y. Retinotomy and foveal translocation for surgical management of subfoveal choroidal neovascular membranes. Am J Ophthalmol 1996;112:613-21.

27. Imai K, de Juan E Jr. Experimental surgical macular relocation by scleral shortening [abstract]. Invest Ophthalmol Vis Sci 1996;37(ARVO Suppl):5116.

28. Imai K, Loewenstein A, de Juan E Jr. Translocation of the retina for management of subfoveal choroidal neovascularization. I. Experimental studies in the rabbit eye. Am J Ophthalmol 1998;125:627-34.

29. Au Eong KG, Pieramici DJ, Fujii GY, Ng EW, Humayun MS, Maia M, et al. Macular translocation: unifying concepts, terminology and classification. Am J Ophthalmol, in press.

30. Pieramici DP, de Juan E Jr, Fujii GY, Reynolds SM, Melia M, Humayun MS, Schachat AP, Hartranft CD. Limited inferior macular translocation for the treatment of subfoveal choroidal neovascularization secondary to age-related macular degeneration. Am J Ophthalmol, in press.

31. Fujii GY, Humayun MS, Pieramici DJ, Schachat AP, Au Eong $K G$, de Juan E Jr. Initial experience of inferior limited macular translocation for subfoveal choroidal neovascularization due to causes other than age-related macular degeneration. Am J Ophthalmol, in press.

32. Fujii GY, de Juan E Jr, Thomas MA, Pieramici DJ, Humayun MS, Au Eong KG. Limited macular translocation for the management of subfoveal retinal pigment epithelial loss following submacular surgery. Am J Ophthalmol, in press.

33. Green WR, Enger C. Age-related macular degeneration: histopathologic studies. The 1992 Lorenz E. Zimmerman lecture. Ophthalmology 1993;100:1519-35.

34. Macular Photocoagulation Study Group. Occult choroidal neovascularization: influence on visual outcome in patients with age-related macular degeneration. Arch Ophthalmol 1996;114:400-12.

35. Harlan JB Jr, Lee ET, Jensen PS, de Juan E Jr. Effect of humidity on posterior lens opacification during fluid-air exchange. Arch Ophthalmol 1999;117:802-4.

36. Fujii GY, Au Eong KG, Jensen PS, Shelley TH, Barnes AC, de Juna E Jr. A new extendable flexible pick for subretinal dissection. Retina, in press.

37. Ng EW, Fujii GY, Au Eong KG, Pieramici DJ, de Juan E Jr. Limited macular translocation for recurrent subfoveal choroidal neovascularization [abstract]. Invest Ophthalmol Vis Sci 2000;41(ARVO Suppl):S540.

38. Fujii GY, Pieramici DJ, Humayan MS, Schachat AP, Reynolds SM, Melia M, et al. Complications associated with limited macular translocation. Am J Ophthalmol, in press.

39. Phillips SJ, Fujii GY, Pieramici DJ, de Juan E Jr. Persistent and recurrent neovascularization after successful macular translocation for subfoveal choroidal neovascularization of age-related macular degeneration [abstract]. Invest Ophthalmol Vis Sci 2000;41(ARVO Suppl):S541. 